\title{
Impedance-based Solid Oxide Fuel Cell testing as scalable and reliable Quality Control tool for cell and batch manufacturing: first findings
}

\author{
Davide Pumiglia ${ }^{1}$, Massimiliano Della Pietra ${ }^{1}$, Andrea Monforti Ferrario ${ }^{1,2, *}$, Stephen McPhail $^{1}$ and Sergii Pylypko $^{3}$ \\ ${ }^{1}$ ENEA, Laboratory of Energy Storage, Batteries and Hydrogen Production \& Use, Department of Energy Technologies and \\ Renewable Sources (TERIN-PSU-ABI), C.R. Casaccia, Via Anguillarese 301, 00123 Rome, Italy \\ ${ }^{2}$ Department of Industrial Engineering and Mathematical Sciences (DIISM), Università Politecnica delle Marche, Via Brecce Bianche \\ 12, 60131 Ancona, Italy \\ ${ }^{3}$ Elcogen AS, Valukoja 23, 11415 Tallinn, Estonia
}

\begin{abstract}
Testing is a necessary step in the manufacturing process of Solid Oxide Fuel Cells (SOFC) to assess the performance of the produced cells before on-field deployment. In this work, the implementation of Electrochemical Impedance Spectroscopy (EIS) and subsequent data elaboration via Equivalent Circuit Modelling (ECM) is evaluated as a viable experimental framework for characterization and Quality Control (QC) of cells or cell batches, complemented to standard polarization-based methods. By performing a statistical analysis of the ECM parameters (representative of each physico-chemical process) the cell and batch operational parameters can be determined and compared. The validity of the proposed methodology is assessed using a wide experimental dataset of a significant number of SOFC samples ( 20 cells from 3 batches - two of which identical and the third with an intentional modification in the manufacturing process in order to validate the proposed methodology) tested in identical conditions. Results show that the impedance-based method provide detailed information in terms of impedance breakdown (anode gas diffusion process resulting the main criticality), as well as confirming the preliminary results obtained from the polarization approach (Batch\#2 showing the lowest total performance and highest uniformity). Highly reproducible intra-batch distributions of the ECM parameters encourage the applicability of such methodology for QC purposes, even with few data collected only in Open Circuit Voltage (OCV) conditions. A relevant deviation of charge transfer and diffusion resistances in Batch\#3 respect to other batches is observed (not visible from the polarization curves), which is reconducted to the modified anode functional layer, opening potential applications of the proposed methodology to assess the impact of targeted modifications of manufacturing methods on specific cell electrochemical performances.
\end{abstract}

\footnotetext{
* Corresponding author: andrea.monfortiferrario@enea.it
} 


\section{Introduction}

Testing is a necessary step in the manufacturing process of Solid Oxide Fuel Cells (SOFC) to assess the performance of produced cells before on-field deployment [1]. Currently, production batches are typically characterized by randomly extracting sample cells from the batch which are tested in terms of output performances at a macro-scale (output power and fuel consumption) in long-term stationary operating conditions and compared with the design parameters. However, such characterization method requires long testing periods to obtain meaningful results (especially for high temperature cells which present a high thermal inertia and require long start-up and stabilization times [2]), representing a challenge for Quality Control (QC) when scaling-up production capacity.

Different SOFC characterization methodologies have been developed, among which polarization-based and impedance-based methods are the most widespread [3]. On the one hand, polarization-based methods are simpler and provide a direct indication of the cell performance under load, however only overall performance indicators can be extracted, and little can be said with respect to any specific process occurring within the cell [4]. On the other hand, albeit providing results that are complex to interpret, impedance-based methods based on Electrochemical Impedance Spectroscopy (EIS) provide more detailed data regarding both the ohmic (ionic conduction) and polarization (charge transfer, mass transfer) processes, as well as providing also information in terms of frequency response of the cell [5-7]. The EIS data can be further deconvoluted via different mathematical formulations, obtaining the breakdown of the impedance response for each physico-chemical process (charge or mass transfer) occurring at the electrolyte or at each electrode [8-10].

Impedance-based testing methods present several similarities with other typical QC techniques. In fact, the performance parameters can be obtained with relatively fast and highly replicable measurements (with respect to endurance testing). Furthermore, since the cell is not loaded, EIS can be almost considered a non-destructive test (with exception of a thermal cycle to reach the operating temperature) $[6,11]$.

In this work, the implementation of EIS and subsequent data elaboration via Equivalent Circuit Modeling (ECM) is evaluated as a viable experimental framework for characterization and Quality Control (QC) of cells or cell batches, respect to standard polarization methods. The applicability of the proposed methodology is analysed applied on an experimental dataset obtained from a significant number of IT-SOFC samples ( 20 cells from 3 production batches) tested in identical experimental conditions (test setup and operating conditions). The quantitative parameters obtained from each characterization technique are analysed statistically to analyse the intra-batch parameter homogeneity - to confirm the applicability of the proposed methodology and subsequently implemented to spot recurrent parameter deviations between batches.

\section{Materials and methods}

Within the qSOFC project, several IT-SOFC button cells provided by Elcogen AS were tested by ENEA for electrochemical characterization. The cells present an Anode Supported Cell (ASC) configuration, with an active surface of $2 \mathrm{~cm}^{2}$ (cathode limited). The thicker supporting anode substrate, together with a very thin layer of electrolyte allows lower operating temperatures (between 650-700 ${ }^{\circ} \mathrm{C}$ ) [12], with respect to other manufacturers. In particular, the SOFC samples were supplied from three different production batches (Batch\#1, Batch\#2 and Batch\#3). For Batch\#1 and Batch\#2 the manufacturing process was identical, whereas for Batch\#3 a slight modification of the anode functional layer morphology was intentionally introduced in the manufacturing process in order to validate the proposed methodology. The modification resulted in a denser anode layer at the interface with the electrolyte with the aim of impacting the hydrogen electro-oxidation charge transfer kinetics due to an increase in the active surface and Triple Phase Boundary (TPB) length.

\subsection{Experimental setup}

The cells were tested in the High Temperature Fuel Cells Operating and Testing Laboratory of ENEA C.R. Casaccia, where two identical test benches were setup. The test bench scheme is illustrated in Figure 1.

The gas distribution system supplies gas from external gas bottles, separate Mass Flow Controllers (MFC Bronkhorst EL-Flow) are installed in each line to control the flow rate of the gases to the anode and cathode compartment. The total flow rates are set to $150 \mathrm{Nml} / \mathrm{min}$ at the anode and $250 \mathrm{Nml} / \mathrm{min}$ at the cathode; which are intentionally abundant with respect to the cell surface to ensure ideal performances [13]. The hydrogen content at the anode fuel gas is controlled by varying the relative flow of $\mathrm{H}_{2}$ and $\mathrm{N}_{2}$ at equal total flow rate, since $\mathrm{N}_{2}$ behaves as an inert gas. Likewise, the oxidant composition can be varied by diluting air with $\mathrm{N}_{2}$. The anode and cathode feeds are supplied to the fuel cell sample through an alumina ceramic housing, placed inside a temperature-controlled furnace (Evenheat Kiln Hot Box) where the cells are mounted vertically, through the use of a high temperature refractory paste (Aremco Ultra-temp 516) which ensures both mechanical support and gas tightness of both anode and cathode chambers.

The electrical interface is composed of an DC electronic power load (Agilent E3634A DC) together with a datalogger (Agilent 34970A) and a LABVIEW-based control system which is used to perform the polarization curves and a separate Impedance Analyzer module (Solartron 1260) coupled with an Electrochemical Interface (Solartron 1287) for the Electrochemical Impedance Spectroscopy (EIS) measurements. The instrumentation is connection to the cell via separate current and voltage wires, attached to current collector meshes to ensure uniform current distribution $(\mathrm{Ni}$ and $\mathrm{Au}$ for anode and cathode side). Separate current and voltage wires reduce voltage perturbation from current flow and allow to perform 4-point impedance measurements. 


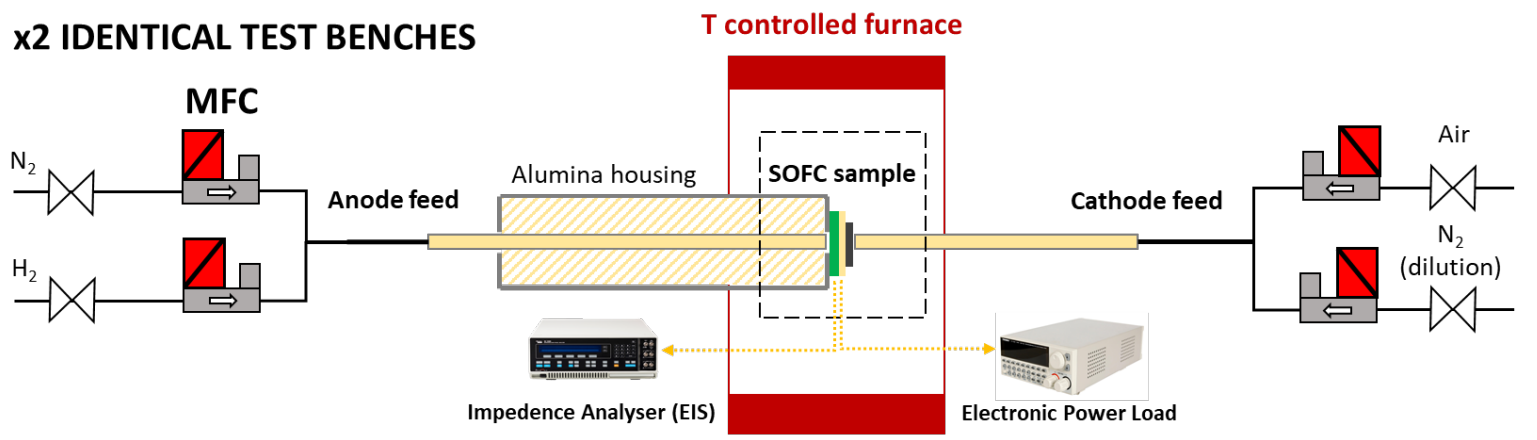

Figure 1. SOFC Test Bench scheme

\subsection{Electrochemical characterization techniques and data elaboration methods}

Each cell is mounted in the experimental setup and characterized with a homogeneous experimental procedure, which is here described.

After following the start-up procedure (controlled $1{ }^{\circ} \mathrm{C} /$ min temperature ramp; gradual reduction of the $\mathrm{NiO}$ at the fuel electrode) each cell is preliminarily characterized in standard conditions $\left(\mathrm{H}_{2} / \mathrm{N}_{2} 50 / 50 \% ; 700\right.$ ${ }^{\circ} \mathrm{C}$ ) with polarization and EIS methods before and after a stabilization period of 50-100 h under a load current of $0.5 \mathrm{~A} / \mathrm{cm}^{2}$, to ensure that stationary conditions have been reached.

Polarization measurements are carried out in galvanostatic mode (step-wise), until reaching $1 \mathrm{~A} / \mathrm{cm}^{2}$ with current in steps of $50 \mathrm{~mA} / \mathrm{cm}^{2}$ or until a cell voltage below $700 \mathrm{mV}$ is reached. After each polarization curve, the cell is stabilized in standard conditions, to remove the produced steam from within the cell which might affect other measurements.

The EIS measurements are carried out in OCV conditions with a frequency range between $100 \mathrm{kHz}-10 \mathrm{mHz}(10$ points per decade) with an applied voltage perturbation amplitude of $10 \mathrm{mV}$ by using the ad-hoc ZPlot/ZView software by Scribner Associates. By analysing the current response, it is possible to map the impedance in the complex plane. The EIS measurements are carried out in OCV conditions to ensure quasi-static operating conditions, avoiding perturbations due to water and heat formation from the electrochemical reaction, which might alter the cell impedance response.

The EIS data can be elaborated with different techniques to obtain even more detailed information on the cell electrochemical parameters:

- By mathematical elaboration, it is possible to transform the complex impedance data into a frequency-dependant function, namely Distribution of Relaxation Times (DRT), whose intensity can be correlated to each separate impedance contribution, attributed to each physico-chemical process occurring at a certain characteristic frequency. An in-house DRT tool developed in MATLAB has been used to derive the DRT function for all EIS measurements [8], implementing adaptive Tikhonov regularization and $\mathrm{K}-\mathrm{K}$ tests to ensure the quality of the output DRT functions.
- By means of Equivalent Circuit Modeling (ECM) method the EIS data can be used to determine quantitative values of the impedance contribution of each physico-chemical process $[5,9,10]$. In fact, a SOFC can be modelled with a well-known equivalent circuit (illustrated in Figure 2), where each circuit element represents a separate physico-chemical process:

The impedance of the electrical connection of the test setup is represented by an ideal inductance $L_{1}$, which provides an impact only at high very frequencies for positive values of the imaginary part of the impedance (not significative in terms of cell response);

$>$ The impedance of the electrolyte, associated to the ionic conductivity/resistivity of the YSZ electrolyte, can be considered constant with frequency and represented by an ideal resistance;

$>$ The charge transfer impedance contributions (for both anode - within the TPB and at the interface and cathode) can be considered as a RC-parallel, whose frequency response is suitable to represent high-frequency phenomena. Instead of an ideal double layer capacitance, a Constant Phase Element $\mathrm{CPE}$ is used to assess non-ideal capacitive behaviour;

$>$ The gas diffusion impedance contributions (for both anode and cathode) can be represented by a semiinfinite Warburg element, which is an equivalent electrical circuit component that models the diffusion process in porous media. The Warburg diffusion element mainly contributes to the frequency response at lower frequencies, which are the characteristic frequencies of the diffusion phenomena.

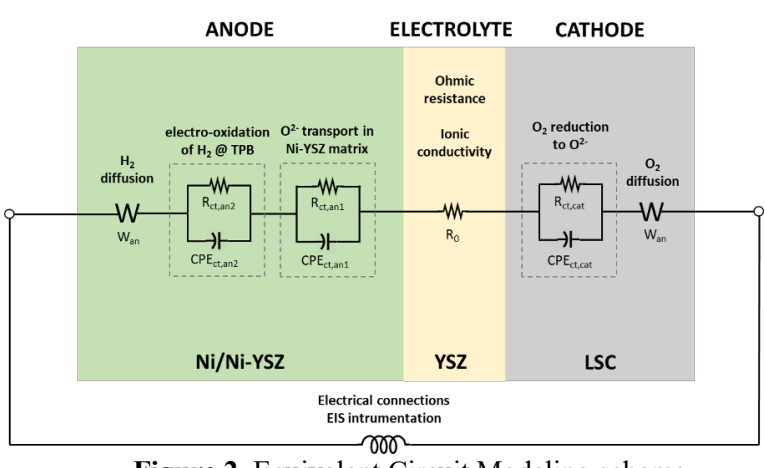

Figure 2. Equivalent Circuit Modeling scheme association of circuit elements to physico-chemical processes 
Given the equivalent circuit topology, the value of each circuit element can be determined via Complex Nonlinear Least Squares (CNLS) fitting method ("Equivalent Circuits" module implemented in ZView software). From the preliminary analysis of the DRT function, initial values for capacitances and resistances can be calculated, rather than being estimated, thus making the fitting more reliable. The numerical algorithm is solved iteratively on gradually wider portions of the frequency range to refine the fitting (according to the preliminary DRT analysis), manually checking for inconsistent values which may arise (since multiple fits can provide the same overall frequency response). The main indicators which are controlled during the fitting process is the $\chi^{2}$ value of the overall fit, the residual (absolute and relative error) of the fit as a function of frequency with respect to the experimental data and the deviation (absolute and relative error) of each circuit element with respect to the initial calculation. An example of the fitting procedure is illustrated in Figure 3.

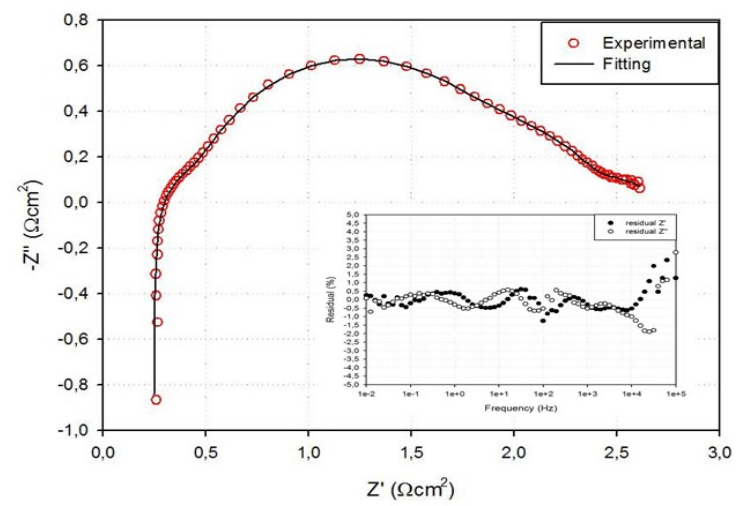

Figure 3. Fitting verification - comparison of the experimental vs. fitted EIS spectra.

With a sufficiently large dataset, the quantitative parameters obtained from each characterization technique (e.g. ASR from polarization, $\mathrm{R}_{0}$ and $\mathrm{R}_{\mathrm{pol}}$ from EIS measurements, circuit elements from ECM) can be analysed statistically to assess the similarity/differences between cells and batches [11,14]. As a preliminary analysis, the average value, distribution (variability assessed in terms of standard deviation $\sigma$ ) and percentile analysis is performed according to classical statistical analysis method.

From a QC perspective, a high degree of similarity within a same batch is an indicator of high manufacturing quality (all cells present homogeneous electrochemical performances); instead, relevant differences could indicate two aspects: i) low manufacturing quality (cells present heterogeneous electrochemical performances) or ii) presence of outliers (differences are recorded only for specific samples). Furthermore, recurrent differences across batches may identify and quantify the impact modifications of the manufacturing process (e.g. modifications in terms of cell design, morphology, architecture and so on), especially in cases when the overall performance (output voltage) is not modified substantially.

\subsection{Experimental campaign design}

Within the qSOFC project 27 cells samples from 3 batches have each been tested, 20 samples were successful in providing coherent results. In Table 1 the tested cells are reported (only the successfully tested cells are included). Overall, a similar number of cell tests were successful on either test bench (12 cells for Test Bench 1 and 8 cells for Test Bench 2) with consistent results, confirming that the test benches can be considered by all means equivalent in terms of performance and do not introduce an experimental offset. More cells from Batch\#2 have been tested due to longer testing time available. At least 5 cells/batch have been tested successfully, to obtain sufficient data for each batch.

Table 1. Tested cell samples and batches*.

\begin{tabular}{|c|c|l|c|}
\hline Batch\#1 & \multicolumn{2}{|c|}{ Batch\#2 } & Batch\#3 ${ }^{* *}$ \\
\hline BC_01_09_TB1 & BC_02_05_TB1 & BC_02_14_TB2 & BC_03_02_TB2 \\
\hline BC_01_11_TB1 & BC_02_07_TB1 & BC_02_15_TB1 & BC_03_03_TB1 \\
\hline BC_01_12_TB2 & BC_02_08_TB2 & BC_02_17_TB1 & BC_03_04_TB2 \\
\hline BC_01_13_TB1 & BC_02_11_TB1 & & BC_03_05_TB1 \\
\hline BC_01_14_TB2 & BC_02_12_TB2 & & BC_03_08_TB2 \\
\hline BC_01_15_TB1 & BC_02_13_TB1 & & \\
\end{tabular}

In this work the analysis is focused only on experimental data collected in standard conditions $\left(\mathrm{H}_{2} / \mathrm{N}_{2} 50 / 50 \%\right.$; $\mathrm{T}=700^{\circ} \mathrm{C}$; stabilized conditions) in the perspective of QC of cell and batch manufacturing.

Thanks to fast EIS measurements, the testing time (including the start-up and stabilization period) can be strongly reduced with respect to performance-based cell characterization.

\section{Results and discussion}

In Section 3.1 the preliminary results from the polarization characterization methods are presented, followed by more detailed results obtained from impedance-based methods in Section 3.2. A statistical analysis of the results is performed to analyse the parameters obtained by each testing method.

Cells with a very high value of $\mathrm{R}_{0}\left(>0.5 \Omega \mathrm{cm}^{2}-\right.$ from EIS spectra) were excluded from the analysed dataset to avoid skewed results, attributing the low performance to testing setup or procedural issues.

It should be noted that results should be assessed on a comparative basis rather than on a quantitative basis, which might be affected by the test setup

\subsection{Preliminary results from polarization methods}

A preliminary analysis of the stabilized polarization curves in standard conditions $\left(\mathrm{H}_{2} / \mathrm{N}_{2} 50 / 50 \% ; \mathrm{T}=700^{\circ} \mathrm{C}\right)$ provides a first indication of the overall electrochemical performance of the tested samples. In Figure 4 the performances of the cells and batches are compared. 

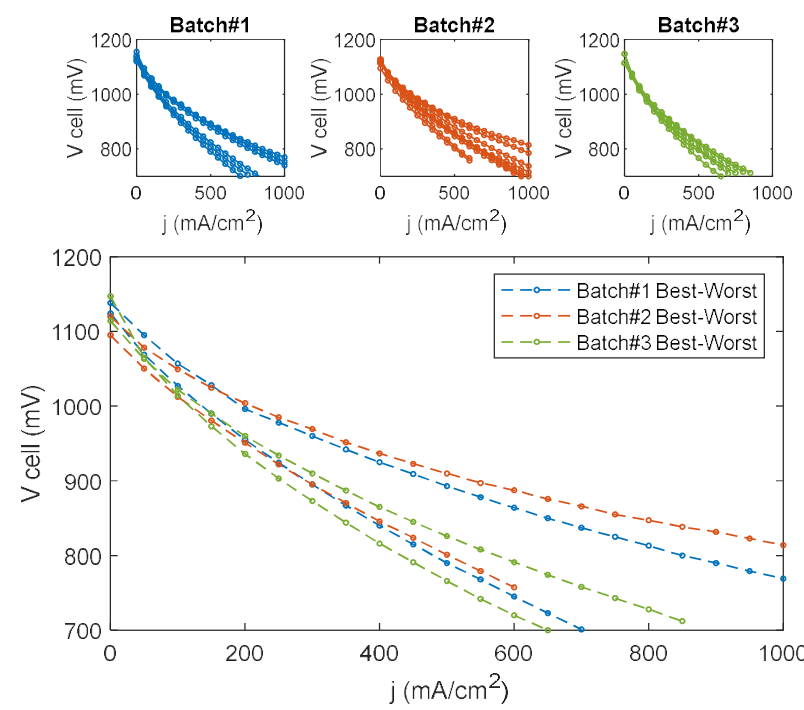

Figure 4. Polarization curves of each cell (top); comparison of best-worst Batch performance range (bottom)

In Figure 5 the OCV and ASR parameters obtained from the IV curves are reported for each Batch. The average OCV and ASR values are summarized in Table 3.

Table 3. Average OCV and ASR parameters obtained by the polarization characterization method.

\begin{tabular}{|c|c|c|}
\hline Parameters & OCV $(\mathrm{V})$ & ASR $\left(\Omega \mathrm{cm}^{2}\right)$ \\
\hline Batch\#1 & 1.13 & 0.44 \\
\hline Batch\#2 & 1.12 & 0.33 \\
\hline Batch\#3 & 1.13 & 0.52 \\
\hline \multirow{2}{*}{ Variability } & $\sigma(\mathrm{OCV})$ & $\sigma(\mathrm{ASR})$ \\
\cline { 2 - 3 } & $(\mathrm{V})$ & $\left(\Omega \mathrm{cm}^{2}\right)$ \\
\hline Batch\#1 & 0.013 & 0.098 \\
\hline Batch\#2 & 0.009 & 0.183 \\
\hline Batch\#3 & 0.018 & 0.056 \\
\hline
\end{tabular}
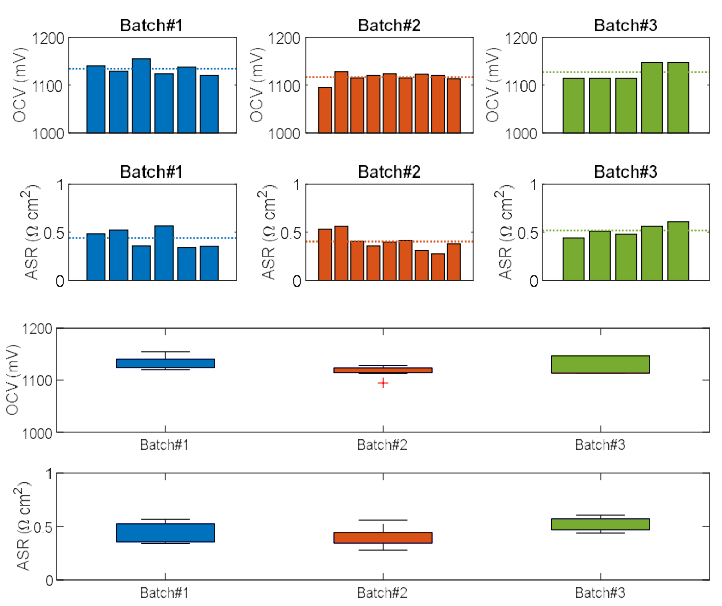

Figure 5. OCV and ASR parameters for each cell (top); Statistical analysis of OCV and ASR for each batch (bottom)

The OCV values are consistently high (between 1.11$1.15 \mathrm{~V}$ - close to the thermodynamic limit [15]), confirming that the gas supply and experimental conditions are suitable for all cells and batches.
By analysing the compared IV curves and electrochemical parameters it is possible to observe that cells from Batch\#2 achieve the highest performances both in terms of average (average ASR equal to $0.33 \Omega$ $\mathrm{cm}^{2}$ ) and best-performing cell (minimum ASR equal to $0.28 \Omega \mathrm{cm}^{2}$ ) performances. However, it can also be seen that Batch\#2 ASR shows also quite a high variability ( $\sigma$ equal to $0.183 \Omega \mathrm{cm}^{2}$ ), comprising also worse performing cells (maximum ASR equal to $0.56 \Omega \mathrm{cm}^{2}$ ), which could be related to the fact that more cells were tested from Batch\#2 respect to other batches, increasing the batch performance variability. Cells from Batch\#1 show intermediate performances, substantially similar to Batch\#2 (average ASR equal to $0.44 \Omega \mathrm{cm}^{2}$ ), with high homogeneity ( $\sigma$ equal to $0.098 \Omega \mathrm{cm}^{2}$ ). Batch\#3, on the other hand, shows a slightly worse average IV performance (average ASR equal to $0.52 \Omega \mathrm{cm}^{2}$ ) close to the lower boundary of Batch\#1 and Batch\#. However, Batch\#3 shows the highest homogeneity ( $\sigma$ equal to 0.056 $\Omega \mathrm{cm}^{2}$ ) of all Batches, meaning that the cells are very performing consistently in the observed performance range. The ASR parameter shows a high variability among cells ( $\sigma$ between $0.05-0.2 \Omega \mathrm{cm}^{2}$ ), denoting that such parameter is not suitable as indicator for QC purposes.

Although the obtained results are coherent with the modification of the manufacturing process for Batch\#3, with the performance-based characterization methods it is difficult to provide additional information, since the output measured signal (IV curve) is a result of a convoluted effect of many different phenomena occurring simultaneously in the cell. In order to obtain more detailed and deconvoluted results, impedance-based methods should be used as described in Section 3.2.

\subsection{Detailed results from Impedance-based methods}

In Figure 6 the complex impedance response for each batch obtained from the EIS measurements in standard stabilized conditions are compared in the Nyquist plane. The impedance contribution can be differentiated between the internal resistance $\mathrm{R}_{0}$ (equal to the intercept of the complex impedance at high frequency) associated to the ionic conduction in the electrolyte and by the electrical resistance of the setup and the polarization resistance $R_{\text {pol }}$ (equal to the intercept of the complex impedance at low frequency), associated to the activation and concentration overpotentials. The summary of the $R_{0}$, $R_{\text {pol }}$ and $R_{\text {tot }}$ values are reported in Table 4 for each batch.

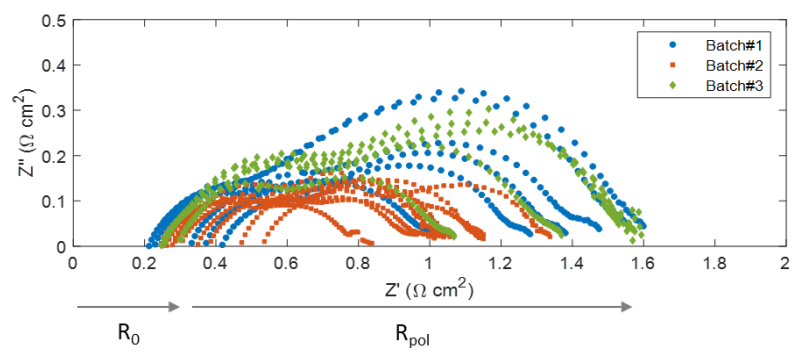

Figure 6. EIS measurements for each cell 
Well-performing cells are typically those showing the lower values of $\mathrm{R}_{0}$ (ca. $0.2 \Omega \mathrm{cm}^{2}$ ), which mainly defines the IV curve slope (however, $R_{0}$ is not exactly equal to the IV curve slope since also the activation and concentration regimes are mutually present). A high value of internal resistance can arise from misalignment between the cathode and the cathodic current collector (because of the vertically oriented cell set up) related to the experimental setup. Nevertheless, a good voltage performance can also be obtained in cells with high $\mathrm{R}_{0}$ if $\mathrm{R}_{\mathrm{pol}}$ is low enough to counterbalance the ohmic losses.

All tested cells show appropriate $\mathrm{R}_{0}$ values (between 0.2 $\left.0.5 \Omega \mathrm{cm}^{2}\right)$ with low internal variability $\left(\sigma<0.1 \Omega \mathrm{cm}^{2}\right)$. Thanks to the similar values of $\mathrm{R}_{0}$ for all batches, the trends previously identified on the ASR are reflected on $\mathrm{R}_{\mathrm{pol}}$ which can be used as a relevant indicator for cell quality (provided that the $\mathrm{R}_{0}$ is within the acceptable range). As previously identified from the analysis of the ASR values, Batch\#2 shows consistently lower $R_{p o l}$ values (between 0.6-0.9 $\Omega \mathrm{cm}^{2}$ ) with respect to Batch\#1 and Batch\#3 (around 0.8-1.2 $\Omega \mathrm{cm}^{2}$ ), highlighting that Batch\#2 is better performing than Batch\#1 and Batch\#3 as well as also presenting the least variability in terms of $\mathrm{R}_{\mathrm{pol}}\left(\sigma<0.1 \Omega \mathrm{cm}^{2}\right)$.

Table 3. Average $\mathrm{R}_{0}, \mathrm{R}_{\mathrm{pol}}$ and $\mathrm{R}_{\text {tot }}$ parameters obtained by the impedance characterization method.

\begin{tabular}{|c|c|c|c|}
\hline \multirow{2}{*}{ Parameters } & $\mathrm{R}_{0}$ & $\mathrm{R}_{\mathrm{pol}}$ & $\mathrm{R}_{\text {tot }}$ \\
\cline { 2 - 4 } & \multicolumn{3}{|c|}{$\left(\Omega \mathrm{cm}^{2}\right)$} \\
\hline Batch\#1 & 0.304 & 1.081 & 1.385 \\
\hline Batch\#2 & 0.347 & 0.717 & 1.064 \\
\hline Batch\#3 & 0.272 & 1.166 & 1.438 \\
\hline \multirow{2}{*}{ Variability } & $\sigma\left(\mathrm{R}_{0}\right)$ & $\sigma\left(\mathrm{R}_{\mathrm{pol}}\right)$ & $\sigma\left(\mathrm{R}_{\text {tot }}\right)$ \\
\cline { 2 - 4 } & \multicolumn{3}{|c|}{$\left(\Omega \mathrm{cm}^{2}\right)$} \\
\hline Batch\#1 & 0.083 & 0.239 & 0.215 \\
\hline Batch\#2 & 0.100 & 0.093 & 0.138 \\
\hline Batch\#3 & 0.026 & 0.220 & 0.227 \\
\hline
\end{tabular}

The variability rate of $R_{\text {pol }}$ (consequently also $R_{\text {tot }}$ ) within each batch is still quite high ( $\sigma$ between $0.1-0.2 \Omega \mathrm{cm}^{2}$ ) denoting that the $R_{\text {pol }}$ is still not suitable as quantitative QC indicator (at least with the tested setup) - unless a very wide variability range is considered. Rather, the use of EIS parameters as QC indicators can be done qualitatively to assess cell and batch quality, with the possibility to discern between $\mathrm{R}_{0}$ and $\mathrm{R}_{\text {pol }}$ (as done in the case of Batch\#2, for which the improvement is ascribed to $R_{\text {pol }}$ rather than $R_{0}$, which presents higher and less consistent values with respect to other batches).

The analyses of the EIS spectra confirm that impedance spectroscopy is a powerful tool for SOFC electrochemical characterization, however it is challenging to determine which specific physicochemical process (charge or mass transfer) changing between cells and batches. To do so an even more accurate analysis of each deconvoluted impedance contribution is required, analysing the ECM elaborated data, which is presented in Subsection 3.2.1.
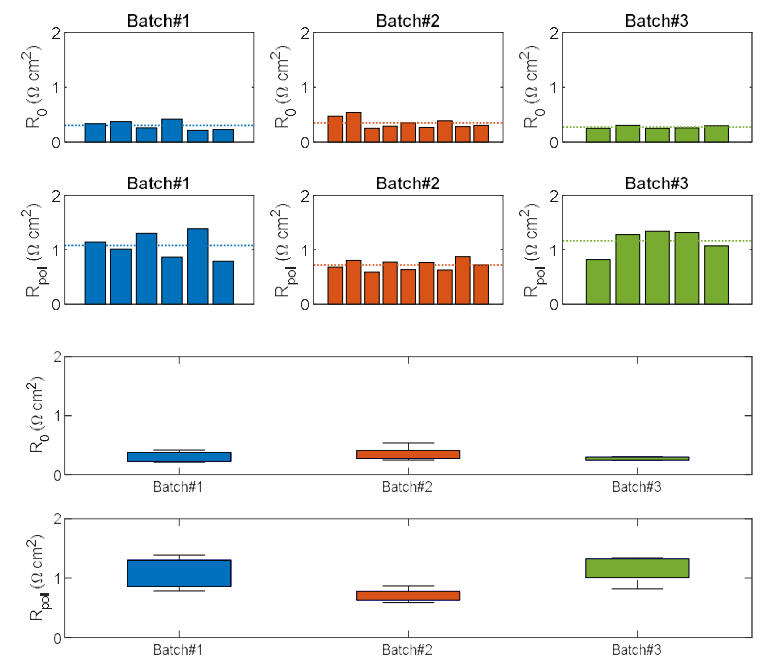

Figure 7. $\mathrm{R}_{0}$ and $\mathrm{R}_{\mathrm{pol}}$ parameters for each cell (top); Statistical analysis of $\mathrm{R}_{0}$ and $\mathrm{R}_{\mathrm{pol}}$ for each Batch (bottom)

\subsubsection{ECM results - Quantitative analysis of deconvoluted Impedance contributions}

Applying the model reported in Figure 2 to the EIS spectra obtained for all the cells in standard conditions, the circuit element parameters - representative of the impedance contribution of each physico-chemical process - can be calculated and compared on a quantitative basis for each cell and batch. The ECM parameters in standard conditions are reported in Figure 8 as well as in Table 5.

The $\mathrm{R}_{0}$ range observed in the ECM results (between 0.2$0.5 \Omega \mathrm{cm}^{2}$ ) is consistent with the one observed in the EIS spectra, confirming that the fitting procedure with respect to this parameter is correctly performed.

Breaking down the polarization resistance it is possible to observe that the main contribution for all batches is related to the mass transport phenomenon at the anode side ( $\mathrm{R}_{\text {diff,an }}$ up to $0.9 \Omega \mathrm{cm}^{2}$ - composing up to $60 \%$ of $\mathrm{R}_{\mathrm{pol}}$ ), followed by the charge transfer mechanism at the anode functional layer (combined $\mathrm{R}_{\mathrm{ct} \text {,an }}$ up to $0.4 \Omega \mathrm{cm}^{2}-$ composing up to $35 \%$ of $\left.\mathrm{R}_{\mathrm{pol}}\right)$. Other processes have a smaller impact in the tested conditions $\left(<0.2 \Omega \mathrm{cm}^{2}\right)$. The main limiting process is the gas diffusion in the anode, which is reasonable considering that the cells are ASC and the gas diffusion paths are longer throughout the thicker bulk anode substrate. On the other hand, the charge transfer mechanism at the anode is slightly limiting (possibly due to $50 \% \mathrm{H}_{2}$ fuel), while charge transfer at the cathode does not represent a limitation (possibly due to highly over-stoichiometric oxidant flow). The cathode diffusive contribution $\mathrm{R}_{\text {diff,cat }}$ is not a limiting factor, showing much lower values than the anodic one $\left(\mathrm{R}_{\text {diff,cat }}<0.15 \Omega \mathrm{cm}^{2}\right)$ for all cells. In fact, the cathode contrarily to the anode - is a thin layer which does not have to provide mechanical support to the cell, therefore the diffusion paths are shorter, allowing the oxidant to easily reach the active layer. However, it must be noted that the cathode diffusive phenomena typically happen at very low frequency (order of $\mathrm{mHz}$ ), where the EIS spectra can present measurement noise which affects the interpretation. 
BATCH \#1
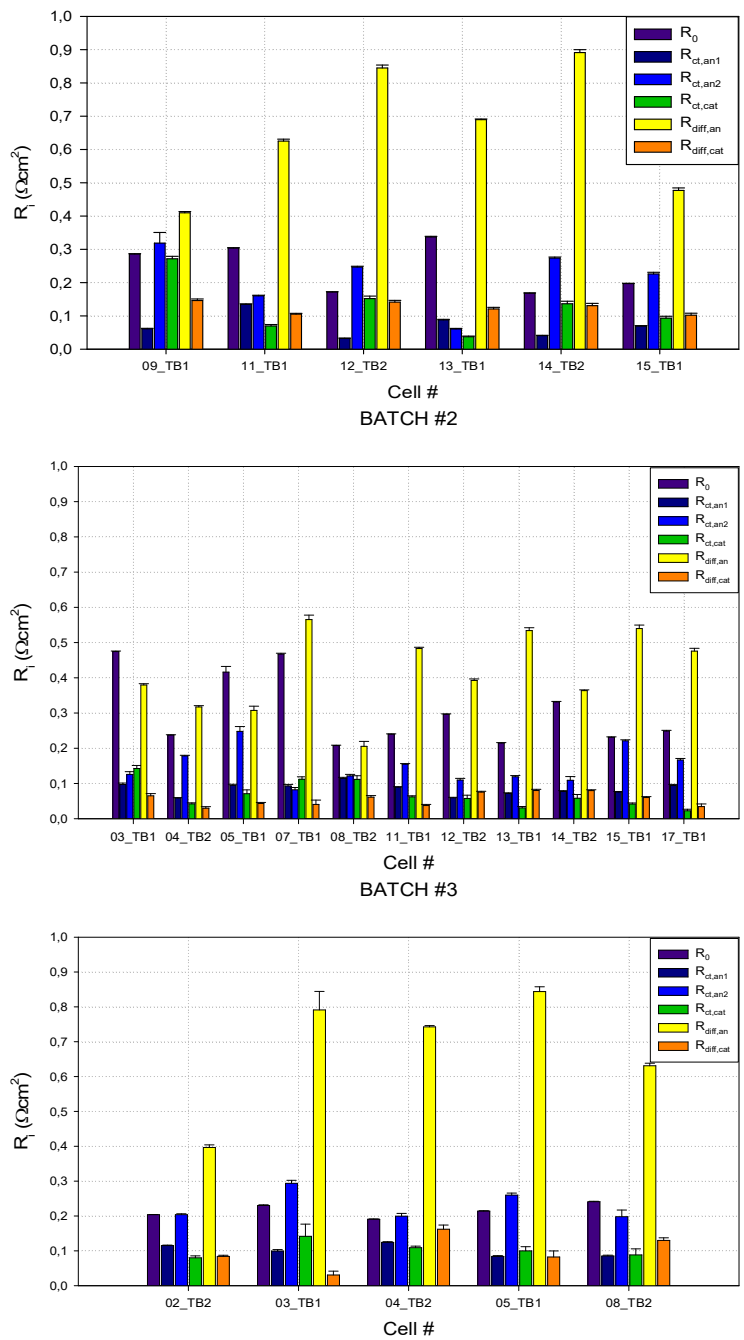

Figure 8. ECM parameters of each cell for Batch\#1 (top); Batch\#2 (middle); Batch\#3 (bottom)

Table 5. Average $\mathrm{R}_{0}, \mathrm{R}_{\text {pol }}$ and $\mathrm{R}_{\text {tot }}$ parameters obtained by the impedance characterization method.

\begin{tabular}{|c|c|c|c|c|c|c|}
\hline \multirow{2}{*}{ Parameters } & $\mathrm{R}_{0}$ & $\mathrm{R}_{\mathrm{ct} \text {,anl }}$ & $\mathrm{R}_{\mathrm{ct} \text {,an2 }}$ & $\mathrm{R}_{\mathrm{ct}, \mathrm{cat}}$ & $\mathrm{R}_{\text {diff,an }}$ & $\mathrm{R}_{\text {diff,cat }}$ \\
\hline & \multicolumn{6}{|c|}{$\left(\Omega \mathrm{cm}^{2}\right)$} \\
\hline Batch\#1 & 0.244 & 0.071 & 0.214 & 0.127 & 0.656 & 0.124 \\
\hline Batch\#2 & 0.295 & 0.086 & 0.148 & 0.063 & 0.430 & 0.057 \\
\hline Batch\#3 & 0.216 & 0.102 & 0.231 & 0.104 & 0.681 & 0.098 \\
\hline \multirow{2}{*}{ Variability } & $\sigma\left(\mathrm{R}_{0}\right)$ & $\sigma\left(\mathrm{R}_{\mathrm{ct}, \mathrm{an} 1}\right)$ & $\sigma\left(\mathrm{R}_{\mathrm{ct}, \mathrm{an} 2}\right)$ & $\sigma\left(\mathrm{R}_{\mathrm{ct}, \mathrm{cat}}\right)$ & $\sigma\left(\mathrm{R}_{\mathrm{diff}, \text { an }}\right)$ & $\sigma\left(\mathrm{R}_{\text {diff,cat }}\right)$ \\
\hline & \multicolumn{6}{|c|}{$\left(\Omega \mathrm{cm}^{2}\right)$} \\
\hline Batch\#1 & 0.074 & 0.037 & 0.092 & 0.083 & 0.193 & 0.018 \\
\hline Batch\#2 & 0.093 & 0.016 & 0.055 & 0.032 & 0.121 & 0.019 \\
\hline Batch\#3 & 0.020 & 0.018 & 0.044 & 0.024 & 0.177 & 0.050 \\
\hline
\end{tabular}

In general, Batch\#2 show slightly lower and less variable ECM results than other batches, indicating better performance and more homogeneity, confirming that the quality of Batch\#2 - in terms of average electrochemical performance - is higher than that from Batch \#1 and Batch\#3.
As previously discussed, the ECM results of Batch\#1 are slightly higher (especially in terms of charge transfer and diffusion resistances) than the ones of Batch\#2. Also, the variability range is slightly higher (average $\sigma$ related to all resistances equal to $0.083 \Omega \mathrm{cm}^{2}$ ) respect to Batch\#2 (average $\sigma$ equal to $0.056 \Omega \mathrm{cm}^{2}$ ), denoting slightly lower manufacturing quality.

Comparing the ECM results of Batch \#3 with the ones from Batch \#2 and Batch\#1, it is possible to highlight that $\mathrm{R}_{\text {ct,an2, as well as }} \mathrm{R}_{\text {diff,an, show a larger contribution to the }}$ overall impedance. The deviations observed from this quantitative comparison are coherent with a modified anode active layer, which mostly affects the related charge transfer mechanism at the TPB. However, the modification seems to have been unsuccessful, resulting in a recurrent increase of $R_{c t, a n 2}\left(0.2-0.3 \Omega \mathrm{cm}^{2}\right.$ vs. 0.1-0.2 $\Omega \mathrm{cm}^{2}$ obtained for other batches). A possible hypothesis could be that the modification in the manufacturing process resulted in a more coarsened (lower TPB length) anode functional layer, less effective from an electrochemical point of view. Moreover, also $\mathrm{R}_{\text {diff,an }}$ seems to increase for all cells (maximum value of around $0.9 \Omega \mathrm{cm}^{2}$ vs. $0.6 \Omega \mathrm{cm}^{2}$ previously obtained for other batches), indicating that the structural modification also affected the gas diffusion process in the anode, possibly in relation to a reduced porosity caused by the denser $\mathrm{Ni}$ grains. On the other hand, a stable value of $\mathrm{R}_{0}$ and a homogeneous batch performance (average $\sigma$ equal to $0.056 \Omega \mathrm{cm}^{2}$ - in alignment with Batch\#2) was obtained, denoting high batch quality, within its measured operating parameters.
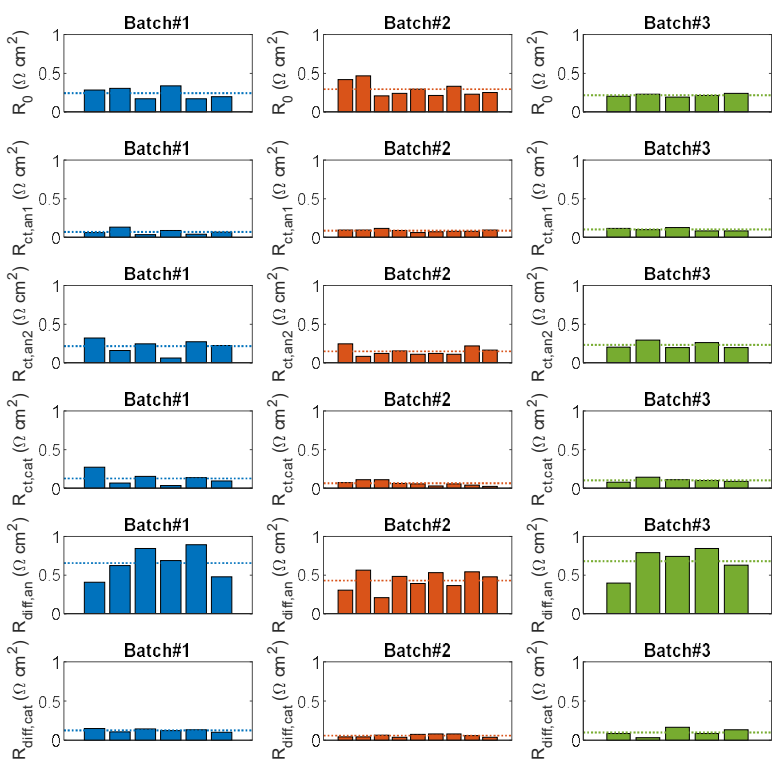

Figure 9. ECM parameters for each cell

Analysing the results under a QC perspective, the intrabatch distribution of the disaggregated ECM parameters (shown in Figure 9 and Figure 10) is much more homogeneous with respect to the parameters that can be obtained from polarization curves (ASR) and from EIS measurements $\left(\mathrm{R}_{0}, \mathrm{R}_{\mathrm{pol}}\right)$. In fact, all ECM parameters -

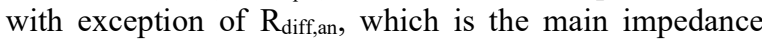
contribution for all cells - are highly replicable 
presenting a very low variability range $\left(\sigma<0.1 \Omega \mathrm{cm}^{2}\right)$. This means that (despite the potential introduction of additional errors caused by the numerical fitting procedure) the ECM parameters are much more suitable to be used as quantitative indicators for QC. In addition, the availability of six parameters instead of one or two global parameters is extremely helpful to analyse similarities/differences of cell and batch performances in relation to specific physico-chemical processes and not only from an overall perspective (different cells could lead to similar aggregated results).

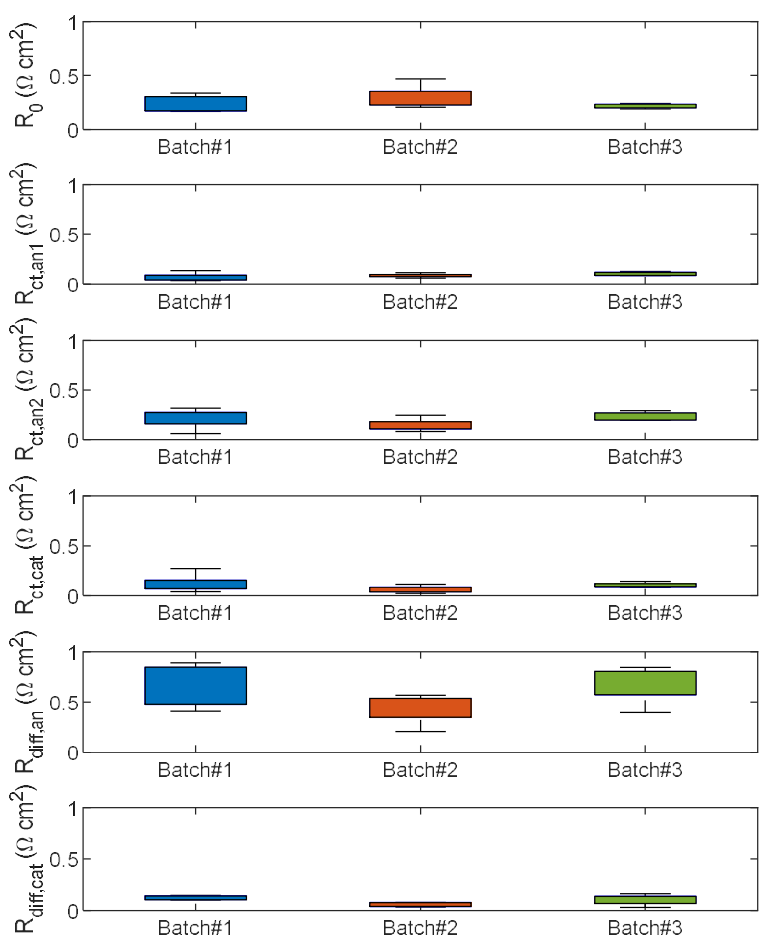

Figure 10. Statistical analysis of the ECM parameters for each Batch

Ideally, from the statistical analysis of a large enough ECM dataset, quantitative threshold limits could be determined and implemented as a scalable and reliable QC tool [11]. Clearly, datasets obtained at laboratory level are insufficient in terms of both data amount (considering equipment availability and testing time constraints) and data quality (considering issues of data replicability), where this methodology can be only demonstrated in principle - as done in this work. Implemented at industry level (with dedicated testing apparatus and implementing automation processes), larger and more consistent ECM datasets could be built, broadening the potential use of such methodology. Advanced data-driven and real-time learning models could be complementarily implemented to improve the dataset generation and management to further enhance the potential of the proposed method as QC tool [14].

\section{Conclusions and future work}

An impedance-based experimental framework has been analysed for the characterization of SOFC cells and batches as an improvement of standard polarization-based methods. A wide experimental dataset composed of 20 IT-SOFC cells from 3 production batches has been used to assess the validity of the proposed methodology.

With respect to the polarization-based method, the impedance-based approach provides detailed and deconvoluted information with respect to each physicochemical process occurring within the cell. Through the calculation of the ECM parameters from EIS data in OCV conditions (which present high intra-batch replicability) it is possible to quantitatively assess the quality and homogeneity of the SOFC operational performances with high reliability, without having to actually operate the cells. By analysing the distribution of the ECM parameters, it is also possible to successfully identify and quantify differences between batches, identifying the impact on electrochemical performances caused by specific modifications of the manufacturing process.

It was possible to assess product quality both within a production batch and between different production batches. Batch\#2 shows lower and more homogeneous $R_{i}$ values, denoting higher quality with respect to other batches, which is reflected in terms of electrochemical performances. Instead, the modified Batch\#3 exhibits a relevant deviation of $R_{c t, a n 2}$ and $R_{\text {diff,an, which is }}$ reconducted to a modified anode functional layer.

Future work will focus on the implementation of a larger experimental dataset, also in different parametric operating conditions as well as improving the statistical analysis to further improve the robustness of the proposed methodology as a reliable QC tool.

This research has been carried out below the EU project qSOFC (Horizon 2020; Grant ID: 735160) which is kindly acknowledged.

\section{References}

1. US Fuel Cell Council. Introduction to Solid Oxide Fuel Cells Button Cell Testing. 2007

2. Mcphail SJ, Aarva A, Devianto H, Bove R, Moreno A.. Int J Hydrogen Energy 2010;36:10337-45.

3. Klotz D, Weber A, Ivers-Tiffée E. Electrochim Acta 2017;227:110-26.

4. Joint Research Centre, JRC Sci Tech Reports 2010.

5. Padinjarethil AK, Bianchi FR, Bosio B, Hagen A. Front Energy Res 2021;9:1-19.

6. Barai A, Uddin K, Dubarry M, Somerville L, McGordon A, Jennings P, et al. Prog Energy Combust Sci 2019;72:1-31.

7. Barfod R, Mogensen M, Klemensø T, Hagen A, Liu YL, Vang Hendriksen P. Proc - Electrochem Soc 2005.

8. Boigues-Muñoz C, Pumiglia D, McPhail SJ, Santori G, Montinaro D, Comodi G, et al. J Power Sources 2015

9. Boigues Muñoz C, Pumiglia D, McPhail SJ, Montinaro

D, Comodi G, Santori G, et al. J Power Sources 2015;

10. Leonide A, Apel Y, Ivers-Tiffee E. ECS Trans 2019.

11. Lang M, Bohn C, Couturier K, Sun X, Mcphail SJ, Malkow T, et al. J. Electr. Soc., 166-15 (2019)

12. Silva-Mosqueda DM, Elizalde-Blancas F, Pumiglia D, Santoni F, Boigues-Muñoz C, McPhail SJ. Appl Energy 2019;235:625-40.

13. Del Zotto L, Monforti Ferrario A, Hatunoglu A, Dell'era A, McPhail S, Bocci E. Energies 2021;14:1-19.

14. Padinjarethil AK, Hagen A. Fuel Cells 2021:1-11.

15. Khotseng L. Thermodyn Energy Eng 2020:1-17. 